\title{
The Star Formation History of Phoenix Dwarf Galaxy using IAC-pop Algorithm
}

\author{
Sebastian L. Hidalgo ${ }^{1}$, Antonio Aparicio ${ }^{2,3}$ \\ and David Martínez-Delgado ${ }^{3}$ \\ ${ }^{1}$ University of Minnesota, Minneapolis, MN, USA \\ email: slhidalgo@astro.umn.edu \\ ${ }^{2}$ Departamento de Astrofísica, Universidad de La Laguna, Tenerife, Canary Island, Spain \\ ${ }^{3}$ Instituto de Astrofísica de Canarias, Vía Láctea, La Laguna, Tenerife, Canary Islands, Spain
}

\begin{abstract}
The Star Formation History (SFH) of the Phoenix dwarf galaxy has been obtained using IAC-star and IAC-pop. The observations are based on WFPC2@HST. The results show a Star Formation Rate (SFR) decreasing with time and that star formation has happened in Phoenix up to 0.5 Gyr ago. The metallicity decrease with the stellar age: $z>0.0007$ for stars younger than 5 Gyr and $z<0.0004$ for stars older than 10 Gyr. Using the SFH as a function of galactocentric radius, the length scale $\alpha_{N}$ of the star density distribution profile has been obtained as a function of the time. The results show that $\alpha_{N}$ decreases smoothly with time, suggesting a sort of shrinking scenario for the evolution of Phoenix.
\end{abstract}

Keywords. galaxies: dwarf, galaxies: stellar content, galaxies: evolution, galaxies: structure

Phoenix is a low surface brightness, dwarf transition galaxy (dSph/dIrr) satellite of the Milky Way (MW), located at a distance of $420 \mathrm{kpc}$ (Hidalgo, Aparicio, \& MartínezDelgado 2007). Previous papers about Phoenix pointed out to an extended population of intermediate-to-old ages with the young, blue stars, concentrated in the central region of the galaxy (Otorlani \& Gratton 1988, Martínez-Delgado, Gallart, \& Aparicio 1999, Held, Saviane, \& Momany 1999, Holtzman, Smith, \& Grillmair 2000, Gallart et al. 2004). There is not HI emission detected in Phoenix, but some HI clouds are in the outskirts (between $4.5^{\prime}$ and $9^{\prime}$ from the center). Phoenix is a ideal candidate to study the stellar populations as a function of the radius: it is close enough to reach the older turn-off using the HST but far away to avoid strongest interactions from the potential well of the MW.

Phoenix was observed using the WFPC2@HST. The observations consist in two fields separated about 2.7'. Images observed by Holtzman et al. 2000 were also used. Total integration times were $23900 \mathrm{~s}$. and $28800 \mathrm{~s}$. for F555W and F814W filters, respectively. The photometry list was obtained using DAOPHOT (Stetson 1994).

To solve the Star Formation History (SFH) of the galaxy, IAC-star (Aparicio \& Gallart 2004) and IAC-pop (Aparicio \& Hidalgo 2007) were used. The solution of the SFH $\psi(t, z)$ as a function of the age and metallicity is shown on the left panel of Fig. 1. Age and metallicity have been divided into 11 and 8 intervals respectively. The volume of each bar on the age-metallicity plane gives the mass that has been ever transformed into stars within the corresponding age-metallicity interval. The Star Formation Rate (SFR) as a function of the age $\psi(t)$ and metallicity $\psi(z)$ are also shown. Star formation activity is present in the galaxy at all ages, with the SFR decreasing with time. Stars as younger as 0.5 Gyr have been detected. The metallicity decreases with the stellar age: $z>0.0007$ for stars younger than 5 Gyr and $z<0.0004$ for stars older than 10 Gyr.

The SFH of Phoenix was derived as a function of the galactocentric radius also. Using this information, the length scale $\alpha_{N}$ of the star density distribution profile as a function 

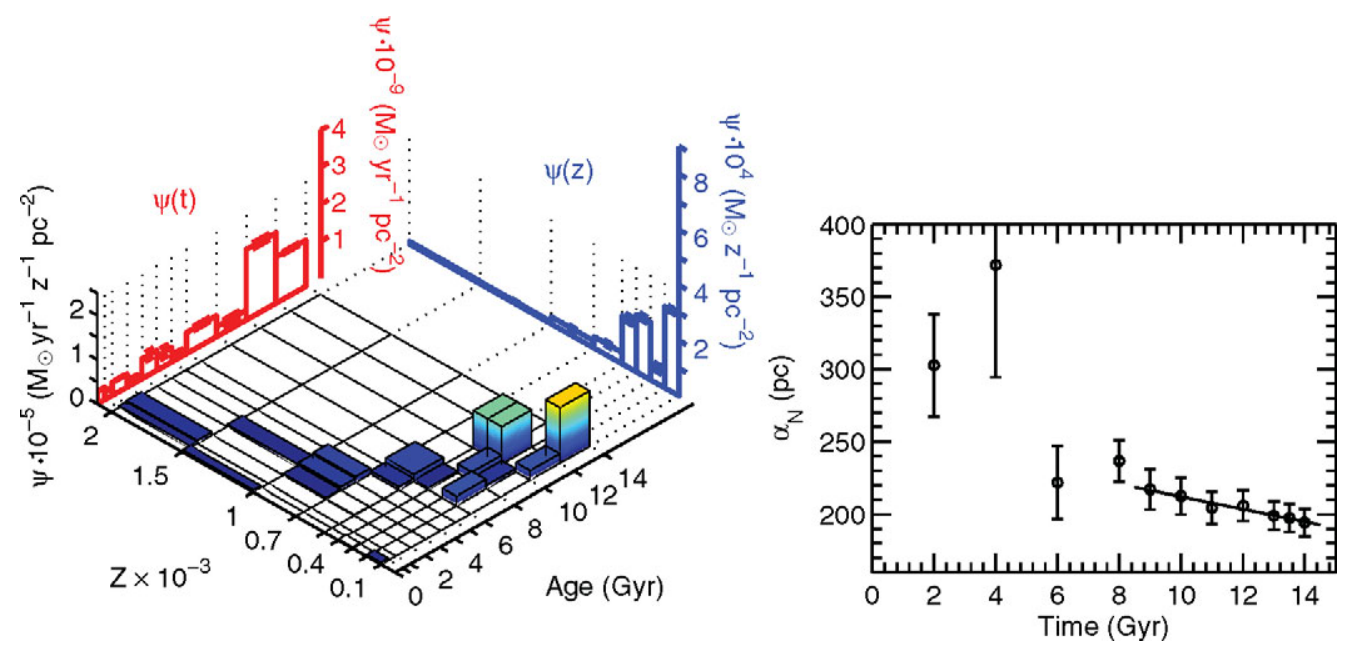

Figure 1. Left panel: The SFH of Phoenix. The SFR as a function of the age $\psi(t)$ and metallicity $\psi(z)$ is also shown. Right panel: Evolution of the scale length of the stellar component of the galaxy as a function of the time.

of time was obtained. It is shown in Fig. 1, right panel. Horizontal axis shows the elapsed time after the formation of the galaxy, assumed to have happened 14 Gyr ago. Vertical axis shows the length scale $\alpha_{N}$ of the star density distribution, in parsecs. It can be observed that the scale length $\alpha_{N}$ decreases with time. A linear function was fitted to the data with $8<\mathrm{t} \leqslant 14 \mathrm{Gyr}$. The result of the fit, shown by a solid line, is $\alpha_{0}=$ $254.91 \pm 15.11(\mathrm{pc})$ and $\gamma=-4.28 \pm 1.23\left(\mathrm{pc} \mathrm{Gyr}^{-1}\right)$, where $\alpha_{0}$ is the length scale at the beginning and $\gamma$, which we call shrinkage scale, gives the direction (negative, i.e., towards the center of the galaxy) and the velocity at which the star formation region decreases in size. This is the shrinking scenario (Hidalgo, Marín-Franch, \& Aparicio 2003). Phoenix is the first galaxy in which this has been carefully tested. Whether this mechanism affects or not to other dwarfs has to be studied.

\section{References}

Aparicio, A., \& Gallart, C. 2004, AJ, 128, 1465

Aparicio, A. \& Hidalgo, S. L. 2007 AJ, submitted

Bertelli, G., Bressan, A., Chiosi, C., Fagotto, F., \& Nasi, E. 1994, AAPS, 106, 275

Gallart, C., Aparicio, A., Freedman, W. L., Madore, B. F., Martínez-Delgado, D., \& Stetson, P. B. 2004, AJ, 127, 1486

Held, E. V., Saviane, I., \& Momany, Y. 1999, AAP, 345, 747

Hidalgo, S. L., Marín-Franch, A., \& Aparicio, A. 2003, AJ, 125, 1247

Hidalgo, S. L., Aparicio, A., \& Martínez-Delgado, D. 2007 ApJ, submitted

Holtzman, J. A., Smith, G. H., \& Grillmair, C. 2000, AJ, 120, 3060

Martínez-Delgado, D., Gallart, C., \& Aparicio, A. 1999, AJ, 118, 862

Ortolani, S., \& Gratton, R. G. 1988, PASP, 100, 1405

Stetson, P. B. 1994, PASP, 106, 250 